\title{
A path optimization signalling protocol for inter-switch handoff in wireless ATM networks ${ }^{1}$
}

\author{
W.S. Vincent Wong ${ }^{2}$, Victor C.M. Leung * \\ Department of Electrical and Computer Engineering, University of British Columbia, 2356 Main Mall, Vancouver, BC, Canada V6T 1Z4
}

\begin{abstract}
In this paper, we study the connection rerouting problem due to inter-switch handoffs. The design objective is to guarantee the bandwidth and delay of a connection during its connection lifetime. We propose to use path extension for each inter-switch handoff, and invoke path optimization whenever one of the potential handoff paths exceed the delay constraint. During path extension, the selection of the extended path is modeled as a bandwidth-delay-constrained path problem. The path optimization signalling protocol ensures that ATM cell sequencing is maintained on the uplink and downlink by buffering cells at the anchor switch and the crossover switch, respectively. (c) 1999 Elsevier Science B.V. All rights reserved.
\end{abstract}

Keywords: Inter-switch handoff; Path extension; Path rerouting; Path optimization; Wireless ATM

\section{Introduction}

Over the past few years, there has been active research in supporting multimedia in wireless networks. With the growing acceptance of ATM (Asynchronous Transfer Mode) as the networking standard in broadband networks, wireless ATM $[14,15,20,21]$ is considered as one of the design alternatives to provide broadband services to the

\footnotetext{
* Corresponding author. Tel.: + 1-604-8226932; Fax: + 1-6048225949; E-mail: vleung@ece.ubc.ca

${ }^{1}$ This work was supported in part by the Natural Sciences and Engineering Research Council of Canada (NSERC) under a Postgraduate Scholarship and Grant No. OGP0044286; and by the Communications Research Centre (CRC), Industry Canada, under the Fessenden Postgraduate Scholarship.

${ }^{2}$ E-mail: vincentw@ece.ubc.ca.
}

mobile users. In order to support mobility in ATM networks, a number of issues have to be resolved [13]. Our work focuses on handoff control in wireless ATM networks [19]. Handoff occurs when a mobile terminal moves from one base station to another. ATM handoff differs from traditional voice handoff in that a mobile user may have several active connections with different bandwidth requirements and quality-of-service (QoS) constraints. The handoff function should ensure that all these ongoing connections are rerouted from one access point to another in a seamless manner. In other words, the design goal is to prevent service disruptions and degradation during and after the handoff process. A key requirement is to have a tight constraint on handoff delays.

There are two kinds of handoff, namely intraswitch handoff and inter-switch handoff. An intraswitch handoff occurs when the mobile terminal 


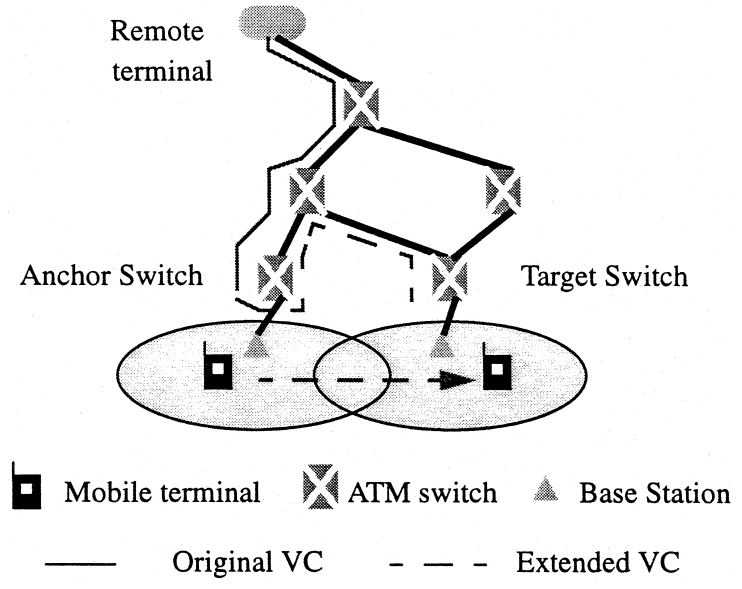

Fig. 1. Path extension scheme.

moves from one base station to another, and both base stations are connected to the same switch. An inter-switch handoff occurs when the mobile terminal moves to another base station which is connected to another switch. Both intra-switch and inter-switch handoff signallings have been specified in Ref. [7]. However, the connection rerouting protocol for inter-switch handoff will not be standardized.

Recently, several connection rerouting protocols to facilitate inter-switch handoff have been proposed in the literature $[1-3,8,18,22-24,29,30]$. Two methods that are based upon partial re-establishment are the path extension ${ }^{3}$ and the path rerouting ${ }^{4}$ schemes. The rationale behind path extension is to extend the original connection to the switch to which the new base station is connected. Referring to Fig. 1 , the switch where the original base station is connected to is usually referred to as the anchor switch, and the switch where the new base station is connected to is called the target switch [3]. During an inter-switch handoff, the path extension method extends the connection from the anchor switch to the target switch. The minimum hop path is usually chosen as the extended path. The path extension scheme is fast and simple to implement. QoS degradations such as cell loss, duplicate cells, and mis-se-

\footnotetext{
${ }^{3}$ Also known as VC extension in [7].

${ }^{4}$ Also known as anchor-based rerouting or dynamic crossover discovery in [7].
}

quence cells do not occur. However, since the extended path is longer than the original one, certain QoS requirements, such as cell transfer delay and cell delay variation, may not be guaranteed after handoff.

Path rerouting can be considered as a generalization of the path extension scheme. In path extension, the anchor switch extends the original connection to the target switch, while in path rerouting, any switch along the original connection can be selected to set up a branch connection to the target switch. Referring to Fig. 2, the switch chosen to perform this function is usually referred to as the crossover switch [22] or the handoff switch [29]. A number of crossover switch discovery algorithms have been proposed in the literature with different performance criteria $[2,10,11,23]$. Despite the fact that path rerouting has the potential of creating an optimal path, the time to determine the location of the crossover switch and the time for branch connection set up may exceed the stringent handoff delay.

In this paper, we study the connection rerouting problem subject to bandwidth and delay constraints. The design objectives are to guarantee the sustainable cell rate and the end-to-end delay of a connection during its connection lifetime. Although the optimal solution of this problem is to compute a new bandwidth-delay-constrained path for each interswitch handoff, the stringent handoff delay and its complexity makes it unfavourable for implementation. As a practical solution, we propose to use path

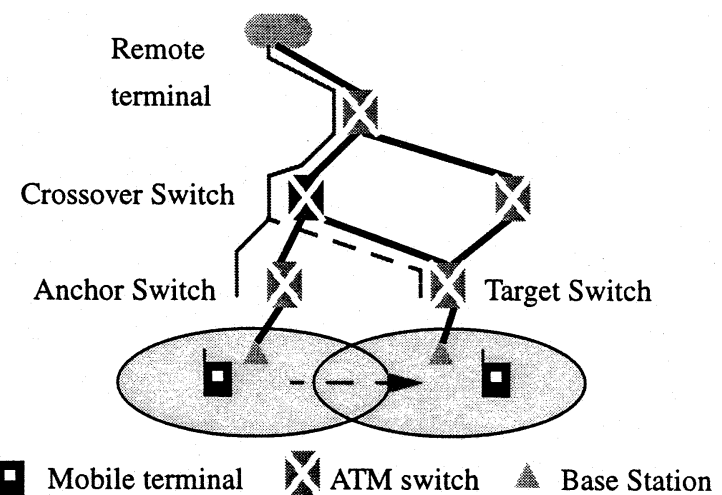

Mobile terminal Original VC

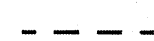

Partial VC 


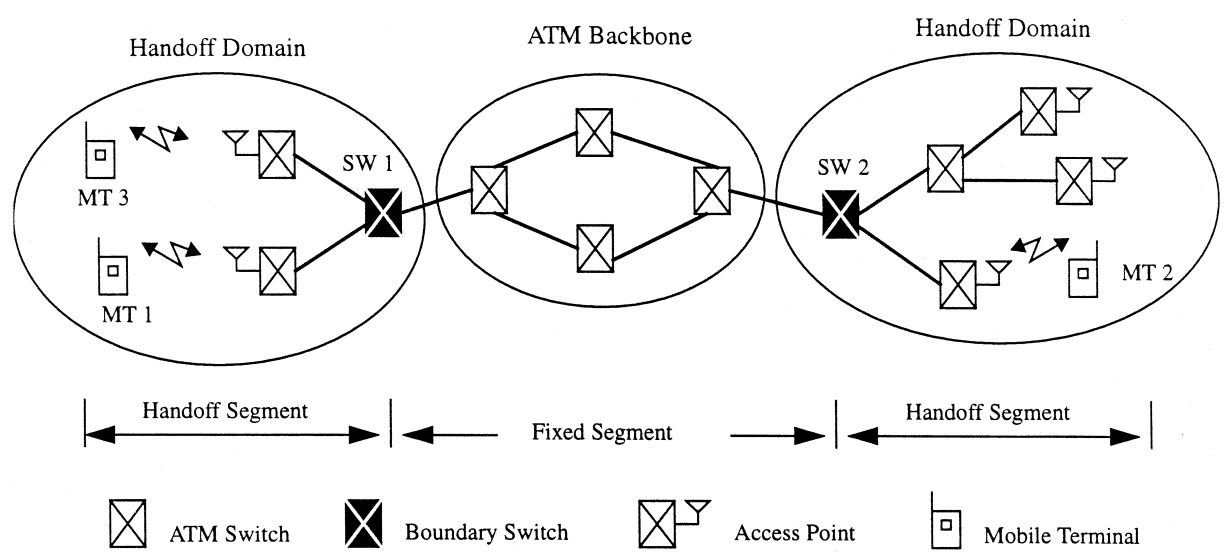

Fig. 3. Handoff reference model.

extension for each inter-switch handoff, and invoke path optimization whenever one of the potential handoff paths exceeds the delay constraint. Although the idea of using path extension and path optimization for inter-switch handoff has been proposed in Ref. [6], in this paper we further define when and how the path optimization is to be performed.

The rest of the paper is organized as follows. The handoff reference model proposed by the ATM Forum is described in Section 2. The network model, along with the definition of the bandwidth-delayconstrained path problem, and a description of the source routing algorithm, are presented in Section 3. Section 4 describes the path extension procedure. Section 5 provides a description of the path optimization algorithm and its signalling protocols. Conclusions and further research directions are presented in Section 6.

\section{Handoff reference model}

The handoff reference model proposed by the ATM Forum [6] is shown in Fig. 3. The model consists of a number of components, including the handoff domain, the fixed and handoff segments, and the boundary switch. The handoff domain is the coverage area where the mobile terminals can move freely with handoff support. The handoff domain consists of a set of access points or base stations, which are then connected to the mobility-enhanced ATM switches.
An end-to-end mobile connection is partitioned into fixed and handoff segments. The handoff segment is part of the connection which can be changed during handoff. A mobile-to-mobile connection has two handoff segments, one for each mobile. The fixed segment, on the other hand, remains unchanged during its connection lifetime. A mobile-to-mobile connection can either have one or no fixed segment.

For each mobile connection, the boundary switch ${ }^{5}$ defines the boundary between the fixed segment and the handoff segment, or the boundary between two handoff segments. The boundary switch is chosen during the call establishment phase and remains unchanged throughout the connection lifetime. If path rerouting is used during inter-switch handoff, depending on the crossover switch discovery algorithms, any switch between the anchor switch and the boundary switch inclusive can be selected as the crossover switch.

To illustrate the relationship between the fixed and handoff segments in a mobile connection, consider the following examples in Fig. 3. For the connection between mobile terminals MT 1 and MT2, the boundary switches are SW 1 and SW 2; the fixed segment is the connection between SW 1 and SW 2; the handoff segments are the connection between

\footnotetext{
${ }^{5}$ Actually this is called the anchor point in [6]. In order to avoid any confusion between anchor point and anchor switch, the name boundary switch is used instead of anchor point in this paper.
} 
SW 1 and MT 1, and the connection between SW 2 and MT 2. For the connection between mobile terminals MT 1 and MT 3, the boundary switch is SW 1; the handoff segments are the connection between SW 1 and MT 1, and the connection between SW 1 and MT 3; there is no fixed segment in this connection.

Note that according to the ATM Forum [6], different handoff domains will be owned and operated by different companies. Although roaming between two handoff domains can be supported, the time for authentication of the mobile users will probably exceed the handoff delay. Thus, handoffs between two handoff domains need not be supported. Hence, path extension and path optimization will be limited to within the handoff domain.

\section{Problem formulation}

Since ATM is connection-oriented, a virtual connection has to be established before communications take place. To setup a connection between two mobile terminals, the following procedures are involved:

- Determine the locations of the mobile terminals;

- Compute the path between the source and the destination;

- Invoke connection admission control along the selected path and make the necessary resource reservations.

The procedures for location management and wireless connection admission control are beyond the scope of this paper. However, how a path is selected during call setup has an impact on the subsequent path extension and path optimization procedures during handoff. In general, an efficient path-finding or routing algorithm should choose a path that has a high chance to guarantee the performance requirements specified by the user. In traditional data networks, the selected path is based upon minimum hop or minimum delay criterion. For realtime applications, the selected path should satisfy multiple QoS constraints, such as bandwidth, delay, and loss probability. Over the past few years, a number of QoS-based routing algorithms have been proposed in the literature $[12,16,17,25]$.
In this paper, the sustainable cell rate and the cell transfer delay are considered as the QoS metrics for path selection. For a mobile-to-mobile connection, the cell transfer delay is the sum of the delays on both wireless and wired links. For path selection in the wired ATM network, only the cell transfer delay between the two edge switches will be considered. Although the path selection process is only based upon two QoS metrics, once a path is determined, other QoS metrics such as cell delay variation and packet loss probability can also be incorporated during the connection admission control phase.

To facilitate our presentation, an ATM network is modeled as a directed, simple connected graph $G=$ $(N, E)$, where $N$ is the set of nodes or ATM switches and $E$ is the set of directed links. Each link $(i, j)$ is assigned some non-negative real numbers $b_{i j}$ and $d_{i j}$ as the residual bandwidth and delay of the link. The residual bandwidth represents the available bandwidth on that link. The delay of a link is the sum of the queueing delay, transmission delay, and the propagation delay over that link.

Given any directed path $p=(i, j, k, \ldots, l, m)$, the bandwidth of path $p$, denoted as Bandwidth $(p)$, is defined as the minimum residual bandwidth of all links on the path

$\operatorname{Bandwidth}(p)=\min \left[b_{i j}, b_{j k}, \ldots, b_{l m}\right]$.

And the delay of path $p$, denoted as $\operatorname{Delay}(p)$, is defined as the sum of all link delays along the path

$\operatorname{Delay}(p)=d_{i j}+d_{j k}+\cdots+d_{l m}$.

During call setup, path extension, and path optimization, the network has to compute a path that can guarantee the sustainable cell rate and the cell transfer delay of the connection. This is a bandwidth-delay-constrained path problem and is formulated as follows.

\subsection{Bandwidth-delay-constrained (BDC) path prob- lem}

Given a graph $G=(N, E)$, a source node $s \in N$, a destination node $d \in N$, a nonnegative bandwidth constraint $B$, and positive delay constraint $D$, com- 
pute the path $p$ from $s$ to $d$ such that the two conditions:

Bandwidth $(p) \geq B$,

$\operatorname{Delay}(p) \leq D$

are satisfied.

The bandwidth constraint $B$ and the delay constraint $D$ correspond to the sustainable cell rate and the cell transfer delay of the connection. The BDC path problem can be solved in polynomial time. A source routing algorithm which solves the above problem, proposed by Wang and Crowcroft [25], is described below. We shall call this algorithm the BDC path algorithm.

The BDC path algorithm involves the following two steps:

1. Create a subgraph $G^{\prime}$ where all the links with residual bandwidth less than the bandwidth constraint $B$ are removed. This ensures all the remaining links have bandwidth greater than or equal to $B$.

2. From the subgraph $G^{\prime}$, use Dijkstra's shortest path algorithm [9] to determine the minimum delay path between the source and the destination. If a path exists and the delay is less than the delay constraint $D$, then a BDC path is found. Otherwise, the network has to renegotiate the bandwidth or delay constraints, or simply reject the connection.

The complexity of step 1 is $\mathrm{O}(E)$ and the complexity of step 2 is $\mathrm{O}\left(N^{2}\right)$. For a simple connected network, $E \leq N^{2}$. Thus, the overall complexity of the BDC path algorithm is $\mathrm{O}\left(N^{2}\right)$.

The algorithm described above is a source routing algorithm, i.e., the path is computed at the source node. If the source maintains the topology information of the entire network, then the route determined by the source is a complete route. However, it is impractical for any nodes to have detailed state information about all nodes and links. Thus in current communications networks, the routing information is stored in a hierarchical manner.

As an example, the Private Network-to-Network Interface (PNNI) protocol [5] is used in ATM networks. In brief, PNNI is a hierarchical routing and signalling protocol. The hierarchy is constructed in a recursive manner by grouping the neighboring nodes at each level into peer groups. Route selection is performed at the source node and at entry border nodes. ${ }^{6}$ The source node computes the entire route to the level of detail visible to it. The entry border nodes continue the route selection and fill in the details of the source route.

Note that if the source node and the boundary switch are located in different peer groups, two issues will arise:

- the path between the source node and the boundary switch seen by the source node is not a complete route;

- the anchor switch may not have sufficient information to determine the location of the crossover switch in a centralized manner.

Hence for simplicity, the path extension and path optimization protocols described in this paper pertain to certain assumptions, which are stated below:

1. The handoff domain is located completely within the PNNI peer group. In this case, for a mobileto-mobile connection between two handoff domains, the two handoff segments are complete routes seen by the corresponding anchor (edge) switches.

2. Each anchor switch maintains a routing information database for the mobile terminals it is currently serving. This database maintains the following information for each mobile connection:

- the mobile terminal identifier (ID);

- the complete route between the anchor switch and the boundary switch;

- the delay constraint between the boundary switch and the anchor switch;

- the current estimated cell transfer delay between the anchor and boundary switches.

3. Each network switch within the handoff domain maintains a VPI / VCI routing information database for the mobile connections it is currently serving. The database stores the following information for each mobile connection:

- the mobile terminal ID;

- the VPI/VCI mapping at the VPI/VCI translation table corresponding to that mobile terminal.

\footnotetext{
${ }^{6}$ A border node is defined as a logical node within a specified peer group, and has at least one link that crosses the peer group boundary.
} 
The first assumption ensures that the anchor switch has sufficient information to determine the location of the crossover switch in a centralized manner. The second assumption ensures that the delay constraint between the anchor switch and the boundary switch is guaranteed by monitoring the cell transfer delay between these two points. The last assumption ensures that for a mobile terminal with a number of active connections, connection rerouting can be performed for all the connections associated with the same mobile terminal.

Notice that instead of having a routing database associated with each ATM switch, a centralized routing database within each handoff domain is also feasible. However, the tradeoffs (e.g., cost, delay, signalling overheads) between a centralized scheme and a distributed scheme require further study. In addition, the concept of global connection identifier (GCID) has also been proposed in Ref. [2]. Each mobile connection is assigned a GCID during its connection lifetime to facilitate the handoff function.

As mentioned in Section 1, an inter-switch handoff consists of two phases. The first phase consists of path extension where the anchor switch extends its connection to the target switch. The second phase consists of path optimization. These two phases are explained in the next section.

\section{Path extension procedure}

The path extension process is invoked whenever an inter-switch handoff occurs. For a backward handoff, ${ }^{7}$ the anchor switch first computes the extended path to the target switch, based on the BDC path algorithm described in Section 3. If the bandwidth and delay constraints are satisfied, connection admission control is then performed along the extended path. After handoff, the target switch becomes the new anchor switch. The estimated cell transfer delay between the boundary switch and the anchor switch is updated. The routing information of

\footnotetext{
${ }^{7}$ In a backward handoff, the mobile terminal sends the handoff request message to the current serving base station. In a forward handoff, the mobile terminal sends the handoff request message to the new (target) base station.
}

the mobile terminal is then transferred to the routing information database associated with the new anchor switch.

The path extension scheme is fast and simple to implement. QoS degradations such as cell loss, duplicate cells and missequence cells do not occur. However, this scheme is not without drawbacks. First of all, data loops will be formed when the mobile terminal moves back to the switch along the original connection. In addition, since the extended path is longer than the original one, certain QoS requirements such as cell transfer delay and cell delay variation, may be violated after handoff.

One way to remove data loops and maintain the delay bound is to compute the path between the anchor switch and the boundary switch again whenever the potential handoff path exceeds the delay constraint. This is the rationale behind the path optimization procedure, which will be described in the next section.

\section{Path optimization procedure}

The path optimization procedure consists of two phases: the path optimization initiation phase and the path optimization execution phase [26]. Path optimization can initiated based on the following criteria:

- QoS-based: Path optimization is triggered when the QoS of the mobile connection is violated.

- Network-based: For a mobility enhanced ATM switch, path optimizations for a group of mobile connections are triggered when the traffic load of that switch is greater than a certain threshold.

- Time-based: For each mobile connection, path optimization is triggered at time instants which are independent to the current QoS and the current load of the network (e.g., periodic path optimization [27,28]).

In this paper, only the QoS-based path optimization initiation is considered. Specifically, path optimization is initiated when the delay constraint of the handoff segment has a chance to be violated after the next inter-switch handoff. During the path optimization initiation phase, the anchor switch performs the following functions for each mobile connection:

1. It monitors the cell transfer delay between the anchor switch and the boundary switch. 
2. It computes the extended paths between the anchor switch and all the potential target switches for future inter-switch handoff, based on the BDC path algorithm.

3. The path optimization execution phase is invoked if the delay of one of the potential inter-switch handoff paths exceeds the specified delay constraint.

The above procedures ensure that the delay of the mobile connection is always below the specified delay constraint.

The major steps during the path optimization execution phase involves:

1. Computing a new path between the anchor switch and the boundary switch;

2. Determining the location of the crossover switch;

3. Setting up a new branch connection;

4. Transferring the user information from the old branch connection to the new one;

5. Terminating the old branch connection.

Since the mobile terminal is still communicating over the extended path via the current base station while the path optimization takes place, this gives enough time to perform the necessary functions while minimizing any service disruptions. The details of the path optimization execution phase are explained in the following subsections.

\subsection{Optimal path computation}

Recall from the handoff reference model in Section 2 that for mobile-to-mobile connection, the fixed segment remains unchanged during its connection lifetime. Hence, instead of determining a new endto-end route between the source and the destination, only the computation of the affected handoff segment is necessary. To facilitate the computation of the handoff segment, the cell transfer delay constraint in the wired ATM network is partitioned into three delay bounds, namely, the delay constraint in the fixed segment, and the delay constraints in the two handoff segments (between the boundary switch and the anchor switch). Thus, for a mobile-to-mobile connection, the path optimization procedures of the two handoff segments are independent to each other. During the optimal path computation, the path between the current edge switch and the boundary switch is determined based on the BDC path algorithm described in Section 3.

\subsection{Crossover switch determination}

After the new handoff segment is computed, the anchor switch determines the location of the crossover switch in a centralized manner. Based on the information from the routing information database, the anchor switch compares the original and the new handoff segments to determine the location of the crossover switch. Starting from the boundary switch, the crossover switch is the one where the original and the new handoff segments begin to diverge. As an example, in Fig. 4, where the connection in the upper portion represents the original handoff segment, and the connection in the lower portion represents the new handoff segment, switch SW 2 is the location of the crossover switch. The original and the new handoff segments can be the same if the boundary switch happens to be the crossover switch. In this case, the current handoff segment is the optimal path. Thus, no further path optimization executions are necessary.

\subsection{Connection setup and rerouting}

Once the crossover switch is determined, connection setup between the anchor switch and the crossover switch is performed. Connection setup is done by using the ATM UNI signalling protocol [4]. Referring to Fig. 5, the anchor switch sends a signalling packet CONNECT to the crossover switch to setup the new handoff segment. The crossover switch then replies by sending a CONNECT ACKNOWLEDGE packet to the anchor switch. This message indicates that the setup of the new handoff segment is successful.

Now, there are two handoff segments established between the anchor switch and the crossover switch.

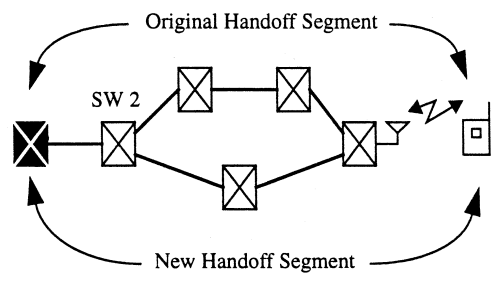

$\bigotimes_{\text {Boundary Switch }} \bigotimes_{\text {ATM Switch }}$

Fig. 4. Crossover switch determination. 


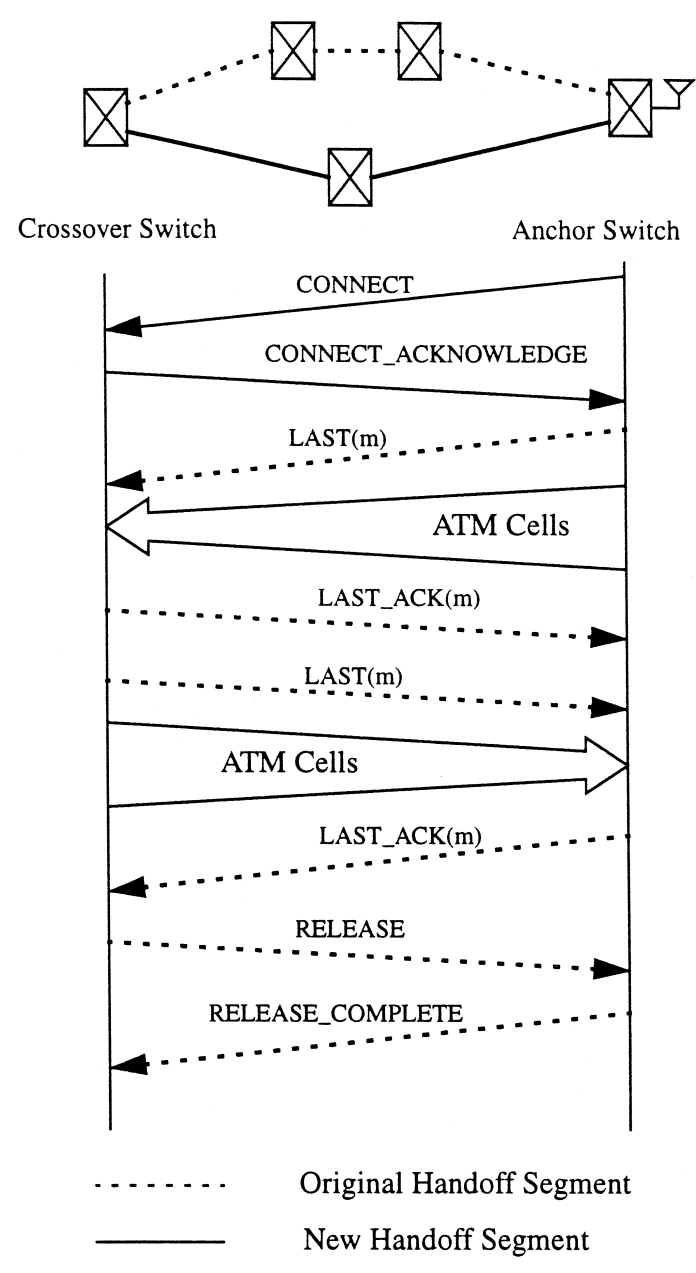

Fig. 5. Connection rerouting signalling message flow.

The connection rerouting protocol has to reroute the data flow from the original handoff segment to the new handoff segment.

In the uplink, the anchor switch sends a message $\operatorname{LAST}(m)$ through the original uplink handoff segment to the crossover switch. This message indicates that no more ATM cells that correspond to mobile terminal $m$ will be transmitted through the original uplink handoff segment. At the same time, the anchor switch begin transmitting ATM cells through the new uplink handoff segment. Since the delay of the new handoff segment is shorter than the delay of the original handoff segment, in order to maintain uplink ATM cells sequencing, the crossover switch will not transmit any ATM cells from the new uplink segment until it has received the LAST $(m)$ message from the original uplink handoff segment. Once the crossover switch receives the $\operatorname{LAST}(m)$ packet, it will begins transmitting ATM cells from the new uplink handoff segment. At the same time, the crossover switch will send a LAST $\operatorname{ACK}(m)$ packet to the anchor switch to acknowledge the reception of the $\operatorname{LAST}(m)$ packet.

Similarly, in the downlink, the crossover switch sends a message $\operatorname{LAST}(m)$ through the original downlink handoff segment to the anchor switch. This message informs the anchor switch that it will receive no more ATM cells correspond to mobile terminal $m$ through the original downlink handoff segment. At this moment, the crossover switch begins to transmit ATM cells through the new downlink handoff segment. To maintain downlink ATM cells sequencing, the anchor switch will not transmit any ATM cells from the new downlink segment until it has received the $\operatorname{LAST}(m)$ message from the original downlink handoff segment. Once the anchor switch receives the LAST $(m)$ message, it will begin transmitting ATM cells from the new downlink handoff segment. At the same time, the anchor switch will send a LAST ACK $(m)$ to the crossover switch to acknowledge the reception of the LAST $(m)$ packet.

Once the connection rerouting process is completed, the crossover switch sends a signalling packet RELEASE to terminate the original handoff segment between the crossover switch and the anchor switch. The RELEASE COMPLETE packet indicates that the original handoff segment has been released. The path optimization process is now completed.

The disruption delay due to connection rerouting, denoted as $\tau$, is bounded by the delay difference between the original and the new handoff segments. If cell loss is to be avoided, buffers can be used at the crossover switch and anchor switch to temporarily store the cells arriving, respectively, from the new uplink and downlink handoff segments, until the respective $\operatorname{LAST}(m)$ messages are received, whereupon the buffered cells and any new cells arriving in the new handoff segments are forwarded in the proper sequence. For symmetric traffic, the buffer size at the anchor switch and the crossover switch is bounded by $\tau R_{\text {peak }}$, where $R_{\text {peak }}$ is the peak cell rate of the connection. 


\section{Conclusions}

Path optimization after each inter-switch handoff may be necessary if the rerouted path is not optimal. In this paper, we have considered the scenario where path extension is used for each inter-switch handoff, and path optimization is invoked whenever the delay of one of the potential handoff paths exceed the delay constraint. The path optimization signalling protocol ensures that uplink and downlink ATM cell sequencing is maintained by buffering at the anchor switch and the crossover switch.

The path optimization process described in this paper is based upon delay constraint criterion. Further work will include the comparisons between other schemes such as network-based or periodic updates. In addition, in this paper we have only considered the scenario where the handoff domain is located within the PNNI peer group. In the situation that the handoff domain consists of a number of peer groups, the design of efficient centralized or distributed crossover switch algorithm remains a research issue.

\section{References}

[1] A.S. Acampora, M. Naghshineh, An architecture and methodology for mobile-executed hand-off in cellular ATM networks, IEEE Journal on Selected Areas in Communications 12 (8) (1994) 1365-1375.

[2] A. Acharya, J. Li, D. Raychaudhuri, Primitives for Location Management and Handoff in Mobile ATM Networks, ATM Forum Contribution 96-1121, August 1996.

[3] B.A. Akyol, D.C. Cox, Signaling alternatives in a wireless ATM network, IEEE Journal on Selected Areas in Communications 15 (1) (1997) 35-49.

[4] The ATM Forum Technical Committee, ATM User-Network Interface (UNI) Specification Version 3.1, September 1994.

[5] The ATM Forum Technical Committee, Private Network-toNetwork Interface Specification Version 1.0 (PNNI 1.0), af-pnni-0055.000, March 1996.

[6] The ATM Forum Wireless ATM Working Group, Baseline Text for Wireless ATM Specifications, BTD-WATM-01.07, April 1998.

[7] The ATM Forum Wireless ATM Working Group, Draft Baseline Text for Wireless ATM Capability Set 1 Specification, BTD-WATM-01.08, July 1998.

[8] B.A.J. Banh, G.J. Anido, E. Dutkiewicz, Handover re-routing schemes for connection oriented services in mobile ATM networks, Proc. IEEE INFOCOM'98, San Francisco, CA, 1998, pp. 1139-1146.
[9] D. Bertsekas, R. Gallager, Data Networks, 2nd ed., PrenticeHall, Englewood Cliffs, NJ, 1992.

[10] R.R. Bhat, R. Gupta, Framework for Dynamic COS Discovery in Wireless ATM, ATM Forum Contribution 98-0005, February 1998.

[11] R.R. Bhat, Extension to Backward COS Discovery (BCD) Approach, ATM Forum Contribution 98-0231, April 1998.

[12] R. Guerin, A. Orda, QoS-based routing in networks with inaccurate information: Theory and algorithms, Proc. IEEE INFOCOM'97, Kobe, Japan, 1997, pp. 75-83.

[13] L. Van Hauwermeiren, L. Vercauteren, A. Saidi, T. Van Landegem, Requirements for mobility support in ATM, Proc. IEEE GLOBECOM'94, San Francisco, CA, 1994, pp. 16911695.

[14] IEEE Communications Magazine, Special Issue on Introduction to Mobile and Wireless ATM, 35 (11) (1997).

[15] IEEE Journal on Selected Areas in Communications, Special Issue on Wireless ATM, 15 (1) (1997).

[16] W.C. Lee, M.G. Hluchyi, P.A. Humblet, Routing subjected to quality of service constraints in integrated communications networks, IEEE Network 9 (4) (1995) 44-55.

[17] D. Lorenz, A. Orda, QoS routing in networks with uncertain parameters, Proc. IEEE INFOCOM'98, San Francisco, CA, 1998.

[18] R. Ramjee, T.F. La Porta, J. Kurose, D. Towsley, Performance evaluation of connection rerouting schemes for ATM-based wireless networks, IEEE/ACM Transactions on Networking 6 (3) (1998) 249-261.

[19] K. Rauhala, Requirements for Mobile ATM Handover, ATM Forum Contribution 96-989, August 1996.

[20] D. Raychaudhuri, N.D. Wilson, ATM-based transport architecture for multiservices wireless personal communication networks, IEEE Journal on Selected Areas in Communications 12 (8) (1994) 1401-1414.

[21] D. Raychaudhuri, L. Dellaverson, M. Umehira, J. Mikkonen, T. Phipps, J. Porter, C. Lind, H. Suzuki, Charter, Scope, and Work Plan for Proposed Wireless ATM Working Group, ATM Forum Contribution 96-0530, April 1996.

[22] C.-K. Toh, Performance evaluation of crossover switch discovery algorithms for wireless ATM LANs, Proc. IEEE INFOCOM'96, San Francisco, CA, 1996, pp. 1380-1387.

[23] C.-K. Toh, Crossover switch discovery for wireless ATM LANs, ACM Journals on Mobile Networks and Applications 1 (2) (1996) 141-165.

[24] M. Veeraraghavan, M. Karol, K.Y. Eng, A Combined Handoff Scheme for Mobile ATM Networks, ATM Forum Contribution 96-1700, December 1996.

[25] Z. Wang, J. Crowcroft, Quality-of-service routing for supporting multimedia applications, IEEE Journal on Selected Areas in Communications 14 (7) (1996) 1228-1234.

[26] W.S.V. Wong, V.C.M. Leung, A path optimization signalling protocol for inter-switch handoff in wireless ATM networks, Proc. 1st International Workshop on Wireless Mobile ATM Implementation, Hangzhou, China, April 1998, pp. 92-98.

[27] W.S.V. Wong, H.C.B. Chan, V.C.M. Leung, Performance evaluations of path optimization schemes for inter-switch 
handoffs in wireless ATM networks, Proc. ACM/IEEE MobiCom'98, Dallas, TX, October 1998, pp. 242-251.

[28] W.S.V. Wong, H.C.B. Chan, V.C.M. Leung, Path optimization for inter-switch handoff in wireless ATM networks, in: Proc. IEEE ICUPC'98, Florence, Italy, October 1998, pp. 615-619.

[29] R. Yuan, S.K. Biswas, D. Raychaudhuri, A signaling and control architecture for mobility support in wireless ATM networks, in: Proc. IEEE ICC'96, Dallas, Texas, 1996, pp. $478-484$.

[30] O.T.W. Yu, V.C.M. Leung, Connection architecture and protocols to support efficient handoffs over an ATM/B-ISDN personal communications network, ACM Journal on Mobile Networks and Applications 1 (2) (1996) 123-139.

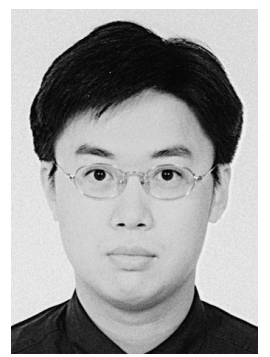

Wai-Shuen Vincent Wong received his B.Sc. degree in electrical engineering from the University of Manitoba in 1994, and his M.A.Sc degree in electrical engineering from the University of Waterloo in 1996. Currently, he is working towards his Ph.D. degree at the University of British Columbia. His research interests include signaling, mobility management and Quality-of-Service (QoS) issues in wireless networks, and QoS-based routing in high speed networks. Mr. Wong is a student member of IEEE.

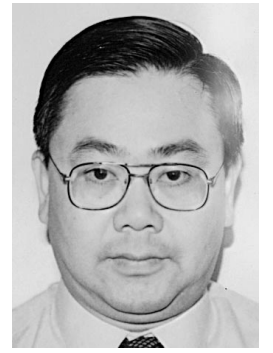

Victor C.M. Leung received the B.A.Sc. (Hons.) degree in electrical engineering from the University of British Columbia (U.B.C.) in 1977, and was awarded the APEBC Gold Medal as the head of the graduating class in the Faculty of Applied Science. He attended graduate school at U.B.C. on a Natural Sciences and Engineering Research Council Postgraduate Scholarship and obtained the Ph.D. degree in electrical engineering in 1981. From 1981 to 1987 ,

Dr. Leung was a senior Member of Technical Staff at Microtel Pacific Research, specializing in the planning, design and analysis of satellite communication systems. He also held a part-time position as Visiting Assistant Professor at Simon Fraser University in 1986 and 1987. In 1988, he was a Lecturer in the Department of Electronics at the Chinese University of Hong Kong. He joined the Department of Electrical Engineering at U.B.C. in 1989, where he is an Associate Professor and a member of the Centre for Integrated Computer Systems Research. He is also a project leader in the Canadian Institute for Telecommunications Research, a Network of Centres of Excellence funded by the Canadian government. His research interests are in the areas of architectural and protocol design and performance analysis for computer and telecommunication networks, with applications in satellite, mobile, personal communications and high speed networks. Dr. Leung is a senior member of IEEE and a voting member of ACM. 